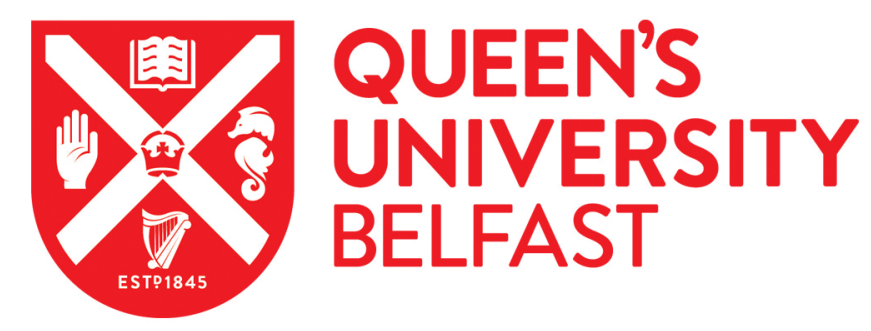

\title{
Chemical Vapor Deposited Hexagonal Boron Nitride as a Scalable Template for High-Performance Organic Field-Effect Transistors
}

Santos, E. (2017). Chemical Vapor Deposited Hexagonal Boron Nitride as a Scalable Template for HighPerformance Organic Field-Effect Transistors. Chemistry of Materials.

https://doi.org/10.1021/acs.chemmater.6b05517

Published in:

Chemistry of Materials

Document Version:

Peer reviewed version

Queen's University Belfast - Research Portal:

Link to publication record in Queen's University Belfast Research Portal

Publisher rights

Copyright 2017 American Chemical Society

This work is made available online in accordance with the publisher's policies. Please refer to any applicable terms of use of the publisher.

\section{General rights}

Copyright for the publications made accessible via the Queen's University Belfast Research Portal is retained by the author(s) and / or other copyright owners and it is a condition of accessing these publications that users recognise and abide by the legal requirements associated with these rights.

Take down policy

The Research Portal is Queen's institutional repository that provides access to Queen's research output. Every effort has been made to ensure that content in the Research Portal does not infringe any person's rights, or applicable UK laws. If you discover content in the Research Portal that you believe breaches copyright or violates any law, please contact openaccess@qub.ac.uk. 


\section{Article}

\section{Chemical Vapor Deposited Hexagonal Boron Nitride as a Scalable Template for High-Performance Organic Field-Effect Transistors}

Tae Hoon Lee, Kwanpyo Kim, Gwangwoo Kim, Hyo Ju Park, Declan Scullion, Leo Shaw, Myung-Gil Kim, Xiaodan Gu, Won-Gyu Bae, Elton J. G. Santos, Zonghoon Lee, Hyeon Suk Shin, Yoshio Nishi, and Zhenan Bao

Chem. Mater., Just Accepted Manuscript • DOI: 10.1021/acs.chemmater.6b05517 • Publication Date (Web): 13 Feb 2017

Downloaded from http://pubs.acs.org on February 20, 2017

\section{Just Accepted}

"Just Accepted" manuscripts have been peer-reviewed and accepted for publication. They are posted online prior to technical editing, formatting for publication and author proofing. The American Chemical Society provides "Just Accepted" as a free service to the research community to expedite the dissemination of scientific material as soon as possible after acceptance. "Just Accepted" manuscripts appear in full in PDF format accompanied by an HTML abstract. "Just Accepted" manuscripts have been fully peer reviewed, but should not be considered the official version of record. They are accessible to all readers and citable by the Digital Object Identifier (DOI®). "Just Accepted" is an optional service offered to authors. Therefore, the "Just Accepted" Web site may not include all articles that will be published in the journal. After a manuscript is technically edited and formatted, it will be removed from the "Just Accepted" Web site and published as an ASAP article. Note that technical editing may introduce minor changes to the manuscript text and/or graphics which could affect content, and all legal disclaimers and ethical guidelines that apply to the journal pertain. ACS cannot be held responsible for errors or consequences arising from the use of information contained in these "Just Accepted" manuscripts. 


\title{
Chemical Vapor Deposited Hexagonal Boron
}

\author{
Nitride as a Scalable Template for High-
}

\section{Performance Organic Field-Effect Transistors}

Tae Hoon Lee $e^{1,2, \dagger}$, Kwanpyo Kim ${ }^{3,4, \dagger}$, Gwangwoo Kim ${ }^{5}$, Hyo Ju Park ${ }^{6}$, Declan Scullion ${ }^{8}$, Leo

Shaw ${ }^{3}$, Myung-Gil Kim ${ }^{3,10}$, Xiaodan Gu ${ }^{3,11}$, Won-Gyu Bae ${ }^{3}$,Elton J. G. Santos ${ }^{3,8,9}$, Zonghoon

Lee $e^{6}$ Hyeon Suk Shin ${ }^{5,7}$, Yoshio Nishi ${ }^{1, *}$, and Zhenan Bao ${ }^{3, *}$

${ }^{1}$ Department of Electrical Engineering, Stanford University, Stanford, CA 94305, U.S.A.

${ }^{2}$ Department of Electrical Engineering, Kwangwoon University, Seoul 139-701, Korea.

${ }^{3}$ Department of Chemical Engineering, Stanford University, Stanford, CA 94305, U.S.A.

${ }^{4}$ Department of Physics, Ulsan National Institute of Science and Technology (UNIST), Ulsan 44919, Korea.

${ }^{5}$ Department of Energy Engineering, Ulsan National Institute of Science and Technology (UNIST), Ulsan 44919, Korea

${ }^{6}$ School of Materials Science and Engineering, Ulsan National Institute of Science and Technology (UNIST), Ulsan 44919, Korea.

${ }^{7}$ Department of Chemistry, Ulsan National Institute of Science and Technology (UNIST), Ulsan 44919, Korea.

${ }^{8}$ School of Mathematics and Physics, Queen's University Belfast, Belfast, BT71NN, United Kingdom. 


\author{
${ }^{9}$ School of Chemistry and Chemical Engineering, Queen's University Belfast, Belfast, BT95AL, \\ United Kingdom. \\ ${ }^{10}$ Department of Chemistry, Chung-Ang University, Seoul,156-756, Korea \\ ${ }^{11}$ Stanford Synchrotron Radiation Light source, SLAC National Accelerator Laboratory, Menlo \\ Park, CA 94025, U.S.A. \\ $\dagger$ These authors contributed equally to this work.
}

\begin{abstract}
Organic field-effect transistors have gained much attention for their potential use in low-cost, large-area, flexible electronics. High-performance organic transistors require a low density of grain boundaries in their organic films and a reduction of charge traps at the semiconductor-dielectric interface for efficient charge transport. In this respect, the role of the dielectric material is crucial because it primarily determines the growth of the film and the interfacial trap density. Here, we demonstrate the use of chemical-vapor-deposited hexagonal boron nitride (CVD h-BN) as a scalable growth template/dielectric for high-performance organic field-effect transistors. The field-effect transistors based on $\mathrm{C}_{60}$ films grown on single-layer CVD h-BN exhibit an average mobility of $1.7 \mathrm{~cm}^{2} \mathrm{~V}^{-1} \mathrm{~s}^{-1}$ and a maximum mobility of $2.9 \mathrm{~cm}^{2} \mathrm{~V}^{-1} \mathrm{~s}^{-1}$ with on/off ratios of $10^{7}$. The structural and morphology analysis shows that the epitaxial, twodimensional growth of $\mathrm{C}_{60}$ on $\mathrm{CVD}$ h- $\mathrm{BN}$ is mainly responsible for the superior charge transport behavior. We believe that CVD h-BN can serve as a growth template for various organic semiconductors, enabling large-area, high-performance flexible electronics.
\end{abstract}


Organic field-effect transistors (OFETs) have been investigated for their high potential in lowcost, large-area flexible electronics. Efficient charge carrier transport in OFETs is critical for extending the scope of their applications. ${ }^{1,2}$ The grain boundaries in polycrystalline organic semiconductor films and trap sites at the organic semiconductor/dielectric interface have detrimental effects on charge transport. ${ }^{3-5}$ Therefore, it is of great importance to reduce the density of grain boundaries by engineering the organic film growth/deposition process and to lower interfacial trap density by optimizing dielectric materials for OFETs.

There have been significant efforts to increase the grain size in organic films. ${ }^{6-8}$ For solutionprocessible organic materials, a variety of processes have been introduced to achieve highly ordered organic films. Notably, using the solution shearing method, we have previously achieved the growth of millimeter-wide and centimeter-long single-crystalline domains of TIPS-pentacene that exhibits high charge carrier mobility. ${ }^{7}$ For vapor deposited organic materials, increasing substrate temperature during deposition is one of the simpler ways for increasing grain sizes. ${ }^{6,8}$ However, fabrication processes at high temperatures above $150^{\circ} \mathrm{C}$ limit the use of some plastic materials required for flexible applications. Since flexible electronics are one of the important target applications for organic semiconductors, a method that can enlarge grain sizes at low temperatures - below $150^{\circ} \mathrm{C}$ - is highly desirable.

Atomically thin 2D materials such as graphene and hexagonal boron nitride (h-BN) have been recently investigated as growth templates for organic films. ${ }^{9-14}$ These $2 \mathrm{D}$ materials are atomically flat without dangling bonds, which can minimize the charge traps at the organic-2D material interface. Moreover, their van der Waals interactions with organic molecules can enable the epitaxial growth of organic films, increasing grain sizes. ${ }^{11-13}$ Indeed, the epitaxial growth of $\mathrm{C}_{60}$, an n-type organic semiconductor, on graphene has been observed. ${ }^{13}$ The low trap density at 
graphene $/ \mathrm{C}_{60}$ interface has been also demonstrated by effective modulation of the Schottky barrier between graphene and $\mathrm{C}_{60} \cdot{ }^{13,15}$ Furthermore, researchers have shown that h-BN can serve as an ideal dielectric layer for graphene devices, with its atomically flat surface, low trap density, and reduction of electron-phonon scattering. ${ }^{16-19}$ The epitaxial growth of other organic molecules such as rubrene and dioctylbenzothienobenzothiophene $\left(\mathrm{C}_{8}\right.$-BTBT) on mechanically exfoliated h-BN flakes has been demonstrated as well; ${ }^{9-11}$ this type of organic film growth enhances structural order, grain size, and charge transport. However, until now, h-BN films obtained from scalable processes, such as chemical vapor deposition (CVD), have not been explored for OFET applications, raising a question as to whether h-BN can be truly suitable for low-cost, large-scale organic electronic manufacturing.

Here, we demonstrate that CVD-grown h-BN is an excellent dielectric to grow organic crystals for electronic applications, especially for OFETs. In this study, we choose $\mathrm{C}_{60}$, a representative n-type organic material, as the channel material. OFETs based on $\mathrm{C}_{60}$ films grown on singlelayer CVD h-BN exhibit an average mobility of $1.7 \mathrm{~cm}^{2} \mathrm{~V}^{-1} \mathrm{~s}^{-1}$ and a maximum mobility of 2.9 $\mathrm{cm}^{2} \mathrm{~V}^{-1} \mathrm{~s}^{-1}$ with on/off ratios of $10^{7}$, showing superior OFET performance compared to that of $\mathrm{C}_{60}$ grown on $\mathrm{SiO}_{2} / \mathrm{Si}$ substrate. Our comprehensive study confirms that the epitaxial twodimensional growth and large grain sizes of $\mathrm{C}_{60}$ on CVD h-BN are mainly responsible for the enhanced charge carrier transport, proving CVD h-BN's suitability as a scalable growth template/dielectric layer. Considering $\mathrm{CVD}$ h-BN has numerous grain boundaries and some surface residues resulted from the transfer process, the obtained device performance can be further improved by optimization of the h-BN CVD and transfer process. Moreover, since the transfer of CVD h-BN does not require high-temperature processing, CVD h-BN will find a 
variety of applications, especially in flexible electronics whose substrates require low temperatures.

\section{Results and discussion}

The large-area growth of h-BN on various metal substrates has been recently demonstrated by $\mathrm{CVD}^{20,21}$ We synthesized single-layer CVD h-BN on Pt foil and transferred it onto a $\mathrm{SiO}_{2} / \mathrm{Si}$ substrate (See Methods for detailed synthesis and transfer process). ${ }^{22}$ The synthesized h-BN generally shows the clean surface morphology with a roughness of $0.17 \mathrm{~nm}$ (Supporting Figure S1) even though it shows some scattered residue resulting from the transfer process. The observed flatness of $\mathrm{h}-\mathrm{BN}$ and its lack of dangling atomic can enable the growth of large $\mathrm{C}_{60}$ grains on h-BN. After the preparation of h-BN template, 20 -nm-thick $\mathrm{C}_{60}$ was evaporated at the rate of $0.2 \AA / \mathrm{s}$ to form a $\mathrm{C}_{60}$ film on h-BN under a vacuum pressure of $2 \times 10^{-6}$ Torr.

The thin film structure of $\mathrm{C}_{60}$ assembly on $\mathrm{h}-\mathrm{BN}$ is investigated using transmission electron microscopy (TEM) imaging and selected area electron diffraction (SAED). There have been recent studies on organic molecular assembly on metal-substrate-supported h-BN by scanning tunneling microscopy. ${ }^{23-25}$ Even though these studies reveal that h-BN can be utilized to obtain well-organized molecular assembly by substrate-molecule interactions, ${ }^{23-25}$ there is no reported study on $\mathrm{C}_{60}$ assembly on h-BN by any molecular imaging tool. We note that the imaging of the packing structures of $\mathrm{C}_{60}$ inside boron nitride nanotubes has been performed by TEM. ${ }^{26}$ In our study, a $\mathrm{C}_{60}$ film assembled on suspended h-BN is investigated by adapting characterization methods similar to our previous study on $\mathrm{C}_{60} /$ graphene. ${ }^{13}$ 
Figure 1 shows TEM images of $\mathrm{C}_{60}$ crystals deposited on h-BN. A low magnification TEM image of the sample shows that $\mathrm{C}_{60}$ evaporated on CVD h-BN has a grain size of $\sim 200 \mathrm{~nm}$, which is larger than that of $\mathrm{C}_{60}$ on amorphous carbon $(\sim 50 \mathrm{~nm})$ (Supporting Figure $\left.\mathrm{S} 2\right)$. This is likely due to the atomically flat nature of h-BN and molecular assembly by $\mathrm{C}_{60} / \mathrm{h}-\mathrm{BN}$ van der Waals interactions. SAED of $\mathrm{C}_{60}$ film on h-BN shows the single-crystal-like signal, which confirms that the grain size of $\mathrm{C}_{60}$ is bigger than $100 \mathrm{~nm}$ - the electron-beam size (Figure 1c,d). Another important feature of TEM is that it allows us to study the in-plane epitaxial alignment of $\mathrm{C}_{60}$ crystals on $\mathrm{h}-\mathrm{BN}$. From the acquired SAED, the relative rotational relationship between $\mathrm{h}-$ $\mathrm{BN}$ and $\mathrm{C}_{60}$ crystal direction can be investigated (Figure 1c,d and Supporting Figure S2). Here, the relative rotation angle $\Theta$ is defined as the angle between the first order diffraction peaks between h-BN and $\mathrm{C}_{60}$ in the reciprocal lattice (Figure 1c,d). $\Theta$ also represents the angle between h-BN zigzag lattice direction and the $\mathrm{C}_{60}$ crystal facet in real space (Figure $1 \mathrm{~b}$ ). The histogram of observed rotational angle $\Theta$ shows two strong peaks at $\Theta=10 \pm 4^{\circ}$ and $\Theta=26^{\circ}$. Our results suggest that there is a mild rotational alignment effect between the lattice of h-BN and the $\mathrm{C}_{60}$ crystallographic direction. High-resolution TEM images of $\mathrm{C}_{60}$ grown on CVD h-BN also clearly reveal highly-ordered local molecular assembly (Figure 1e). The $\mathrm{C}_{60}$ crystals on mechanically exfoliated h-BN flakes shows more pronounced epitaxy with rotational angles at $\Theta=10 \pm 1^{\circ}$ and $\Theta=26^{\circ}$ (Supporting Figure S3). Therefore, the somewhat broadened angular distribution on CVD h-BN, especially around at $\Theta=10^{\circ}$, can be attributed to extrinsic factors such as the polycrystalline nature of CVD h-BN (the average grain size of $\sim 1$ micrometer) and the imperfect surface quality of CVD h-BN since these nucleation sites can induce of non-ideal epitaxial growth of $\mathrm{C}_{60}$ crystals. 
To further understand the observed interfacial alignment, we perform first-principles density functional theory calculations including van der Waals (vdW) dispersion forces (see Methods for details). We initially considered a number of $\mathrm{C}_{60}$ molecular configurations towards $\mathrm{h}$ - $\mathrm{BN}$ surface and calculated their electronic and energetic structures. A computational screening taking into account the relative orientation of atoms at $\mathrm{C}_{60}$ molecule at apex, pentagon, hexagon, and dimer positions resulted in 12 different interface geometries with h-BN (Supporting Figure S4). The adsorption energies were computed at two different levels with and without vdW interactions (Supporting Figure S3), which strikingly show the effect of nonlocal correlations responsible for energies as high as one order of magnitude relative to non-vdW calculations. This result strongly indicates that $\mathrm{vdW}$ interactions play a role in the creation of the interface. Moreover, we also observed a cooperative effect from the interactions of the $\mathrm{C}_{60}$ molecules in the monolayer coverage of $\mathrm{C}_{60}$ on the h-BN surface (Supporting Figure S5). This cooperativeness is responsible for more than $16 \%$ of the calculated binding energy as compared to more dilute systems. An interaction energy at the $\mathrm{C}_{60}$ layer of $-0.52 \mathrm{eV}$ per molecule is observed, which allows further polarization of the interface and extra charge redistribution. The different bonding nature between $\mathrm{C}-\mathrm{C}$ and B-N determined by their electronegativities - B(2.040), $\mathrm{C}(2.551)$, and N(3.041) - also provides an additional ingredient for the stabilization of the interactions.

By taking into account the different configurations of $\mathrm{C}_{60} / \mathrm{h}-\mathrm{BN}$ interface and choosing the most stable structure, we calculate the crystal-direction-dependent adhesion energies (opposite in sign to binding energies) as a function of the rotational angle $\Theta$ (Figure $1 \mathrm{~g}$ ) for the entire set of values. We use the same orientation as Figure $1 \mathrm{~b}$ above, that is, $\Theta=0$ and $\Theta=30^{\circ}$ correspond to zigzag and armchair directions. Our vdW simulations clearly show that configurations near zigzag direction ( 0-2 degrees), 9-12 degrees, and 21 25 degrees have higher stability than nearby 
angular orientations. Experimentally-observe epitaxy angles (Figure 1f and Supporting Figure S3) generally capture the calculated epitaxy energy landscape, in which a large population of samples with an orientation closes to $\sim 10^{\circ}$ are measured. We note that the expected epitaxy near 0 degree is not clearly observed in the experimental data.

The morphology of $\mathrm{C}_{60}$ films evaporated on $\mathrm{CVD}$ h- $\mathrm{BN} / \mathrm{SiO}_{2}$ is also investigated using atomic force microscopy (AFM) as shown in Figure 2. The topography and phase AFM images in Figures $2 \mathrm{a}$ and $2 \mathrm{~b}$ show the terrace structure of $\mathrm{C}_{60}$ on $\mathrm{CVD}$ h-BN, which indicates the twodimensional, layer-by-layer growth mode of $\mathrm{C}_{60}$ on h-BN. Measured step heights between $\mathrm{C}_{60}$ layers are $\sim 0.8 \mathrm{~nm}$, which is consistent with the known thickness of a single layer of $\mathrm{C}_{60}$ (Figure 2f). The two-dimensional growth of $\mathrm{C}_{60}$ on $\mathrm{CVD}$ h-BN is facilitated by the van der Waals interactions between $\mathrm{C}_{60}$ and $\mathrm{CVD}$ h-BN, which was also observed in TEM experiments. In contrast, $\mathrm{C}_{60}$ evaporated on $\mathrm{SiO}_{2}$ shows island-shaped grains as demonstrated in Figure 2c. The apparent grain size of $\mathrm{C}_{60}$ on CVD h-BN is around 100 200 nm, a few times larger than that of $\mathrm{C}_{60}$ on $\mathrm{SiO}_{2}$. Moreover, $\mathrm{C}_{60}$ on $\mathrm{CVD}$ h-BN has better grain connectivity and a more uniform height distribution than $\mathrm{C}_{60}$ on $\mathrm{SiO}_{2}$ as depicted in Figures $2 \mathrm{~d}$ and 2e. The better grain connectivity observed combined with the large grains of $\mathrm{C}_{60}$ on $\mathrm{CVD}$ h-BN indicates the potential for more efficient charge transport in $\mathrm{C}_{60} / \mathrm{h}-\mathrm{BN} / \mathrm{SiO}_{2}$ compared to $\mathrm{C}_{60} / \mathrm{SiO}_{2}$.

Grazing-incidence wide angle X-ray diffraction (GIXD) measurements provide structural information on the morphology of $\mathrm{C}_{60}$ that are grown on $\mathrm{CVD}$ h-BN on a larger area (a few hundred of micrometers). Figure $3 \mathrm{a}$ and $3 \mathrm{~b}$ display the GIXD data for $\mathrm{C}_{60}$ on $\mathrm{CVD}$ h-BN and $\mathrm{C}_{60}$ on $\mathrm{SiO}_{2}$, respectively. The diffraction patterns show a strong reflection on the meridian at $\mathrm{q}_{\mathrm{z}} \sim$ $0.76 \AA^{-1}$, which corresponds to the (111) plane of a face-centered cubic (fcc) lattice. This indicates that the (111) faces of $\mathrm{C}_{60}$ fcc grains are well-aligned and parallel to CVD h-BN 
surface, confirming the epitaxial growth of $\mathrm{C}_{60}$ mediated by van der Waals interactions. Other observed peaks are also consistent with the two-dimensional closed packed structure of $\mathrm{C}_{60}$ film. ${ }^{27}$ On the other hand, isotropic diffraction rings in Figure $3 \mathrm{~b}$ indicate that $\mathrm{C}_{60}$ fcc grains are randomly oriented on $\mathrm{SiO}_{2}$.

The coherence length of $\mathrm{C}_{60}$ crystal is calculated using the Scherrer formula ${ }^{28}$ and the full width at half-maximum $(\mathrm{FWHM})$ values of the $(11 \overline{1})$ reflection at $\mathrm{q}_{\mathrm{xy}}=0.73 \AA^{-1}$ and $\mathrm{q}_{\mathrm{z}}=0.24 \AA^{-1}$. The calculated coherence length for $\mathrm{C}_{60}$ on $\mathrm{CVD}$ h-BN is $\sim 47 \mathrm{~nm}$, whereas the coherence length of $\mathrm{C}_{60}$ on $\mathrm{SiO}_{2}$ is $\sim 14 \mathrm{~nm}$. The larger coherence length of $\mathrm{C}_{60}$ on h-BN as compared to that on $\mathrm{SiO}_{2}$ confirms our TEM and AFM results.

We now discuss the electrical characteristics of OFETs based on $\mathrm{C}_{60}$ on CVD h-BN. Figure 4a shows a schematic of $\mathrm{C}_{60}$ OFET prepared on CVD h-BN. A representative transfer curve of FETs incorporating $\mathrm{C}_{60}$ on $\mathrm{CVD}$ h-BN is shown in Figure $4 \mathrm{~b}$. The highest observed mobility is $2.9 \mathrm{~cm}^{2} \mathrm{~V}^{-1} \mathrm{~s}^{-1}$ (Figure $4 \mathrm{~b}$ ). The very high average mobility of $1.7 \mathrm{~cm}^{2} \mathrm{~V}^{-1} \mathrm{~s}^{-1}$ is achieved using $\mathrm{C}_{60}$ on CVD h-BN as shown in the histogram of measured mobility values in Figure 4c. Transfer curves for $\mathrm{C}_{60}$ on $\mathrm{SiO}_{2}$ are given for comparison to $\mathrm{C}_{60}$ on $\mathrm{CVD}$ h-BN as shown in Figures $4 \mathrm{~d}$ and 4e. The charge carrier mobility of $\mathrm{C}_{60}$ on $\mathrm{CVD}$ h-BN is 40 times higher compared to that of $\mathrm{C}_{60}$ on $\mathrm{SiO}_{2}$, which shows the average value of $0.04 \mathrm{~cm}^{2} \mathrm{~V}^{-1} \mathrm{~s}^{-1}$. Since the mobility in organic fieldeffect transistors is very sensitive to the surface roughness and traps on a dielectric layer, the high mobility suggests that CVD h-BN is an excellent dielectric and growth template for $\mathrm{C}_{60}$.

We emphasize that this is the first demonstration of organic semiconductor growth and device fabrication on large-area CVD h-BN. Previously, various surface treatments of dielectric substrate, such as functionalization by self-assembled molecular layer, have been utilized to 
improve the morphology/grain size of deposited $C_{60}$ crystals and related device performance. ${ }^{29-32}$ The best reported mobility values of $\mathrm{C}_{60}$ transistors on these functionalized surfaces range from 2 to $5 \mathrm{~cm}^{2} \mathrm{~V}^{-1} \mathrm{~s}^{-1}$, where the highest value $\left(\sim 10 \mathrm{~cm}^{2} \mathrm{~V}^{-1} \mathrm{~s}^{-1}\right)$ was obtained with solution-processed isolated $\mathrm{C}_{60}$ single crystals. ${ }^{29-32}$ When we consider $\mathrm{C}_{60}$ transistors composed of fully-covered $\mathrm{C}_{60}$ film, the observed maximum mobility value of $2.9 \mathrm{~cm}^{2} \mathrm{~V}^{-1} \mathrm{~s}^{-1}$ using CVD h-BN is comparable to the highest reported mobility values. Currently, the CVD synthesis process for h-BN is not as optimized as that for graphene, and thus the grown h-BN layer has smaller grain sizes (the average grain size of $\sim 1$ micrometer) and a noticeable density of residues, resulting in the hysteresis in the transistor operation (Supporting Figure S6). The device performance is expected to improve further as the h-BN synthesis and transfer processes are optimized.

\section{Conclusion}

In conclusion, we demonstrate that $\mathrm{CVD}$ h-BN is an excellent dielectric layer for $\mathrm{C}_{60}$ deposition and FET applications. The epitaxial relationship between $\mathrm{C}_{60}$ and h-BN is studied in detail using TEM characterization and first-principles calculations. The large sizes and crystal orientation of $\mathrm{C}_{60}$ grains on $\mathrm{CVD}$ h-BN are investigated with $\mathrm{AFM}$ and GIXD. Furthermore, we report highperformance field-effect transistors based on $\mathrm{C}_{60}$ on $\mathrm{CVD}$ h-BN. The high mobility values reaching $2.9 \mathrm{~cm}^{2} \mathrm{~V}^{-1} \mathrm{~s}^{-1}$ suggest that $\mathrm{CVD} \mathrm{h}-\mathrm{BN}$ is a promising dielectric for organic semiconductor devices. Given its low temperature requirements, CVD h-BN is expected to find more general use for flexible devices that require low temperature process. 


\section{Methods}

h-BN Synthesis and Transfer. h-BN was synthesized using chemical vapor deposition. ${ }^{22}$ Ammonia borane was heated at $130^{\circ} \mathrm{C}$ to be decomposed into borazine, hydrogen, and polyiminoborane. The borazine and hydrogen gas was flowed onto $\mathrm{Pt}$ foil in a furnace at $1100^{\circ} \mathrm{C}$. Then, borazine is decomposed and forms single layer h-BN on the Pt foil. For the transfer process, PMMA was spin coated onto the h-BN/Pt foil and cured. The PMMA/h-BN/Pt foil was dipped into a $1 \mathrm{M}$ aqueous solution of $\mathrm{NaOH}$ and used as the cathode of an electrolysis cell. The water reduction at the cathode generates $\mathrm{H}_{2}$ bubbles between PMMA/h-BN and the Pt foil, which then detach the PMMA/h-BN from the Pt foil. The separated PMMA/h-BN was then transferred onto $300 \mathrm{~nm} \mathrm{SiO}_{2} / \mathrm{Si}$ substrates for transistor fabrication. After the transfer, PMMA was removed in acetone, and then residual PMMA was further removed by heating at $400^{\circ} \mathrm{C}$ for $2 \mathrm{~h}$ under $\mathrm{H}_{2} / \mathrm{Ar}$ atmosphere. For comparison study, BN powers from Momentive Performance Materials were mechanically exfoliated to produce thin h-BN flakes on $\mathrm{SiO}_{2} / \mathrm{Si}$ wafer. The samples were calcinated at $450^{\circ} \mathrm{C}$ for 1 hour to remove possible tape residue on the surface of h-BN flakes. For preparation of TEM grids with CVD h-BN, PMMA-covered CVD h-BN was transferred onto Quantifoil holey carbon TEM grids and PMMA was removed in aceton. In the case of mechanically exfoliated h-BN samples, the flakes on $\mathrm{SiO}_{2} / \mathrm{Si}$ wafer were transferred to TEM grids by the direct transfer method. ${ }^{33}$

$\mathrm{C}_{60}$ Film Preparation and Au Evaporation. Before $\mathrm{C}_{60}$ evaporation, TEM grids with CVD h$\mathrm{BN}$ were pre-annealed in air at $200^{\circ} \mathrm{C}$ for $30 \mathrm{~min}$ to minimize the possible adsorbates on the $\mathrm{h}$ BN surface. After the pre-annealing process, TEM grids were attached onto the evaporator substrate with Kapton tape. 300-nm $\mathrm{SiO}_{2} / \mathrm{Si}$ substrates were also fixed onto the evaporator substrate for device fabrication. 20-nm-thick $\mathrm{C}_{60}$ films were thermally evaporated on both h- 
BN/TEM grids and device substrates at the substrate temperature of $110^{\circ} \mathrm{C}$ with an evaporation rate of $0.2 \AA^{-1}$. Au electrodes (40 nm thick) were thermally evaporated through a shadow mask with a channel length and width of 50 um and 1000 um respectively.

TEM Characterizations. TEM imaging and selected area electron diffraction (SAED) acquisition were performed with a FEI Titan Cube G2 60-300 operated at $80 \mathrm{kV}$ with an image aberration corrector. Extra SAED acquisition was performed using a JEOL 2100F, which is operated at $200 \mathrm{kV}$.

GIXD Characterization. GIXD images were collected in reflection mode with a 2-D area detector (MAR-345; pixel size of $150 \mu \mathrm{m}, 2,300 \times 2,300$ pixels) and the sample in a helium atmosphere at beamline 11-3 of the Stanford Synchrotron Radiation Lightsource. The sample to detector distance was $400 \mathrm{~mm}$, which was calibrated using a $\mathrm{LaB}_{6}$ polycrystalline standard. The incidence angle was $0.12^{\circ}$. The X-ray wavelength was $0.9758 \AA$, corresponding to a beam energy of $12.7 \mathrm{keV}$. Before performing quantitative analysis on the images, the data was corrected for the theta-dependent image distortion caused by the planar detector surface. Numerical integration of the diffraction peak areas was performed with the software WxDiff. The overall resolution in the GIXD experiments was about $0.01 \AA^{-1}$.

Electrical Characterization. The device measurement was performed inside a nitrogen glove box using a Keithley 4200-SCS semiconductor parameter analyzer. The saturation mobility was extracted from the slope of the transfer curve of $\mathrm{I}_{\mathrm{DS}}-\mathrm{V}_{\mathrm{GS}}$, using $\mathrm{I}_{\mathrm{DS}}=\mu \mathrm{WC}_{\mathrm{ox}}\left(\mathrm{V}_{\mathrm{G}}-\mathrm{V}_{\mathrm{T}}\right)^{2} / 2 \mathrm{~L}$, where $\mu$ is the mobility, $\mathrm{W}$ is the channel width, $\mathrm{L}$ is the channel length, $\mathrm{C}_{\mathrm{ox}}$ is the gate capacitance per unit area, and $\mathrm{V}_{\mathrm{T}}$ is the threshold voltage. 
Theoretical Calculations. Calculations were based on ab initio density functional theory using the SIESTA method ${ }^{34}$ and the VASP code. ${ }^{35,36}$ The generalized gradient approximation ${ }^{37}$ along with the DRSLL ${ }^{38,39}$ functional was used in both methods, together with a double- $\zeta$ polarized basis set in SIESTA, and a well-converged plane-wave cutoff of $500 \mathrm{eV}$ in VASP. We used a Fermi-Dirac distribution with an electronic temperature of $\mathrm{k}_{\mathrm{B}} \mathrm{T}=20 \mathrm{meV}$. Additional details are provided in the Supporting Information. 

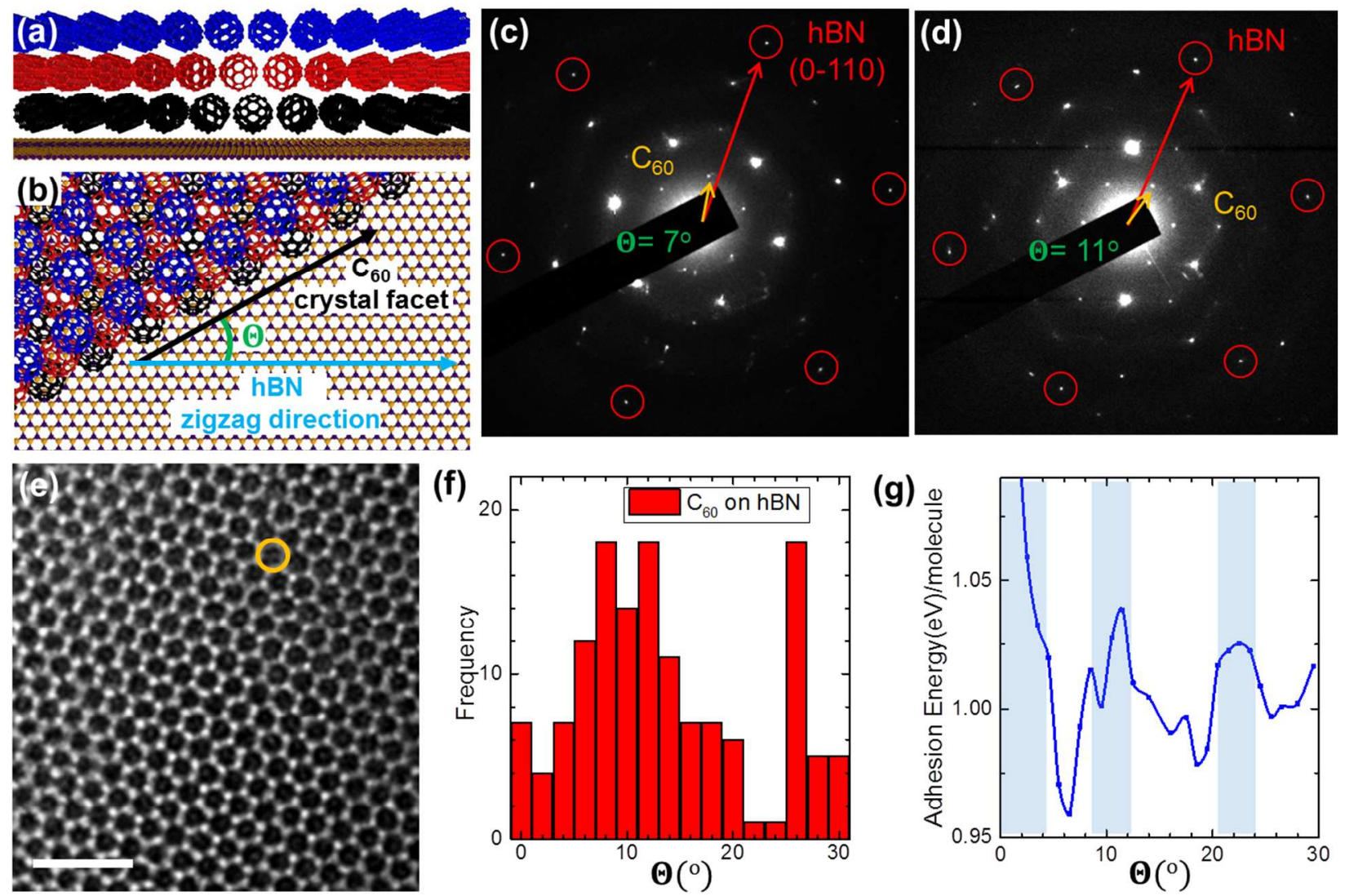

Figure 1. Orientation alignment of $\mathbf{C}_{60}$ crystals on CVD h-BN membrane. (a) A schematic side-view of $\mathrm{C}_{60}$ film on h-BN membrane. (b) Top-view of $\mathrm{C}_{60}$ crystal on h-BN. The relative angle $\Theta$ between h-BN zigzag lattice direction and $\mathrm{C}_{60}$ crystal facet is used for labelling epitaxial alignment. (c,d) Selected area electron diffraction of $\mathrm{C}_{60}$ film on h-BN. The single-crystal-like $\mathrm{C}_{60}$ diffractions are observed. (e) High-resolution TEM image of $\mathrm{C}_{60}$ crystal prepared on h-BN. The marked circle indicates the size and location of $\mathrm{C}_{60}$ molecule. Scale bar, $2 \mathrm{~nm}$. (f) Histogram of observed relative rotational angle of $\mathrm{C}_{60}$ crystal on $\mathrm{h}-\mathrm{BN}$. (g) Calculated orientation-dependent adhesion energy (opposite sign to binding energies) of $\mathrm{C}_{60}$ on h-BN. Colored regions indicate stable configurations with local maximum adhesion energy. 

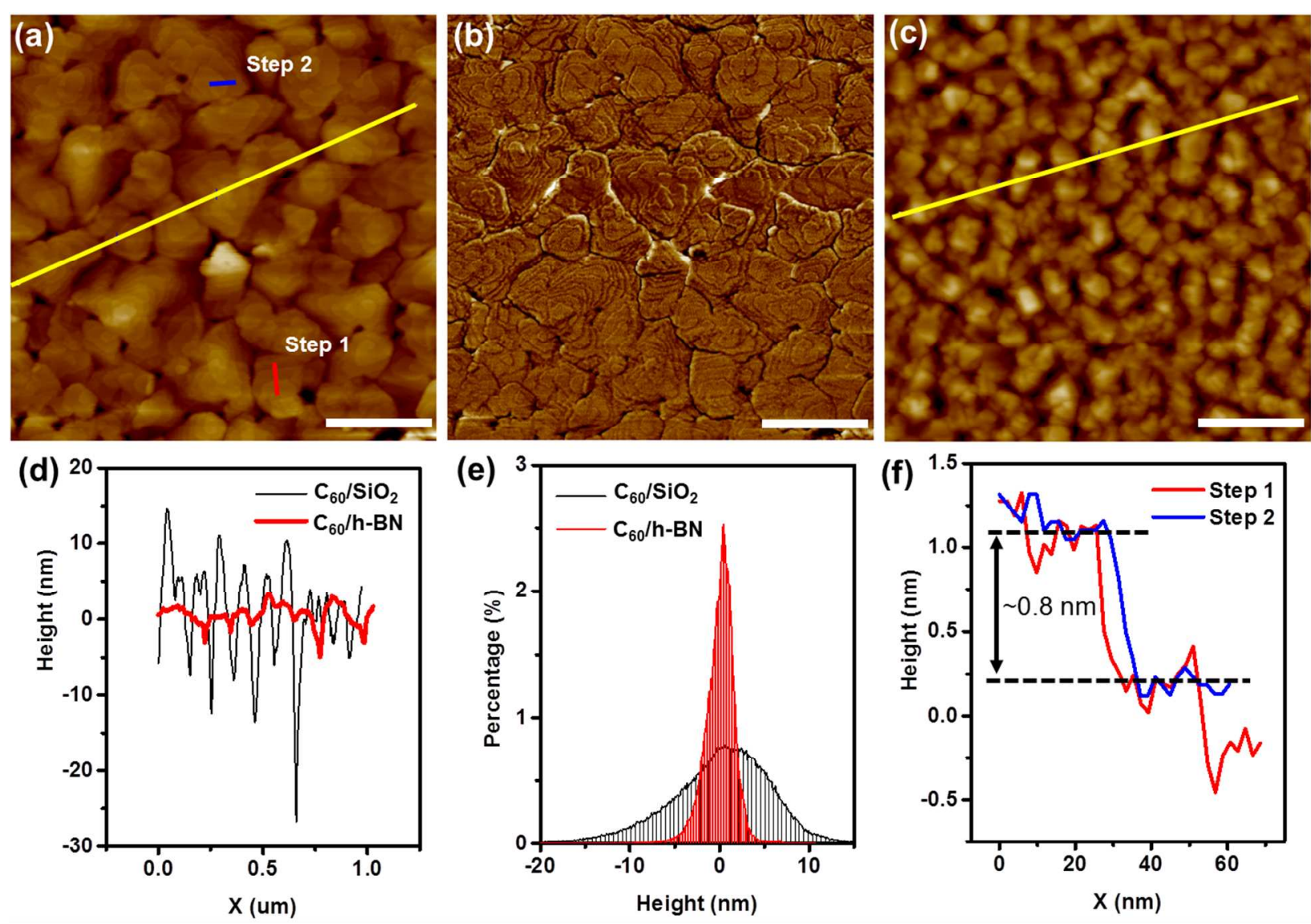

Figure 2. Two-dimensional growth of $\mathbf{C}_{60}$ on CVD h-BN. (a) AFM height and (b) phase images for $\mathrm{C}_{60}$ on CVD h-BN. Clear terrace structure and large $\mathrm{C}_{60}$ grains are observed. Scale bar, $250 \mathrm{~nm}$. (c) AFM height image for $\mathrm{C}_{60}$ on $\mathrm{SiO}_{2} \cdot \mathrm{C}_{60}$ on $\mathrm{SiO}_{2}$ has a small grain size and no terrace structure. Scale bar, $250 \mathrm{~nm}$. (d) Comparison of AFM cross-section images for $\mathrm{C}_{60}$ on h$\mathrm{BN}$ and $\mathrm{C}_{60}$ on $\mathrm{SiO}_{2}$ along marked yellow lines. Height profile for $\mathrm{C}_{60}$ on h-BN is more uniform than that for $\mathrm{C}_{60}$ on $\mathrm{SiO}_{2}$. (e) Histogram of the height distribution. (f) Step heights between $\mathrm{C}_{60}$ layers. Observed step heights are around $0.8 \mathrm{~nm}$, which is consistent with the known $\mathrm{C}_{60}$ singlelayer thickness value. 


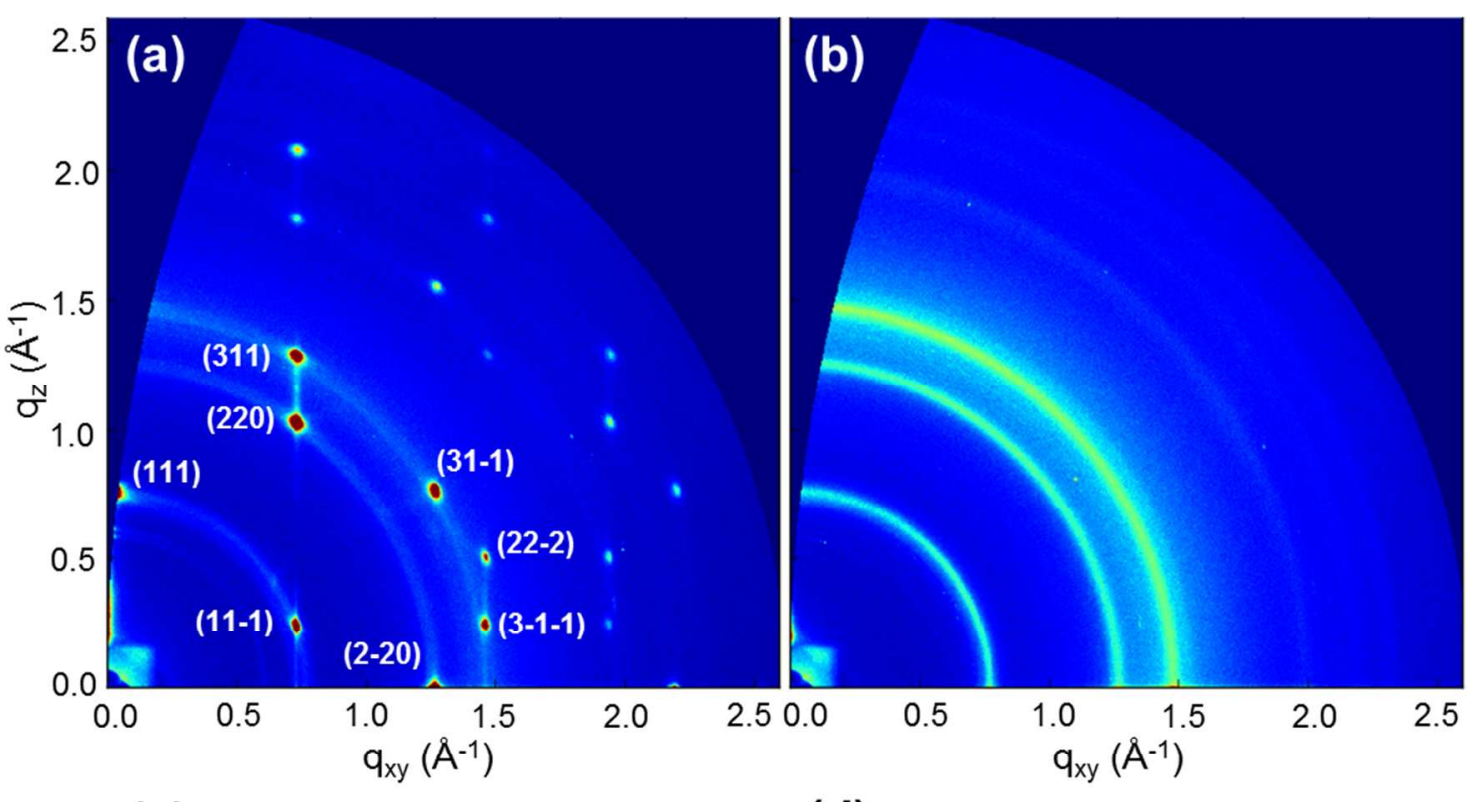

(c)

(d)
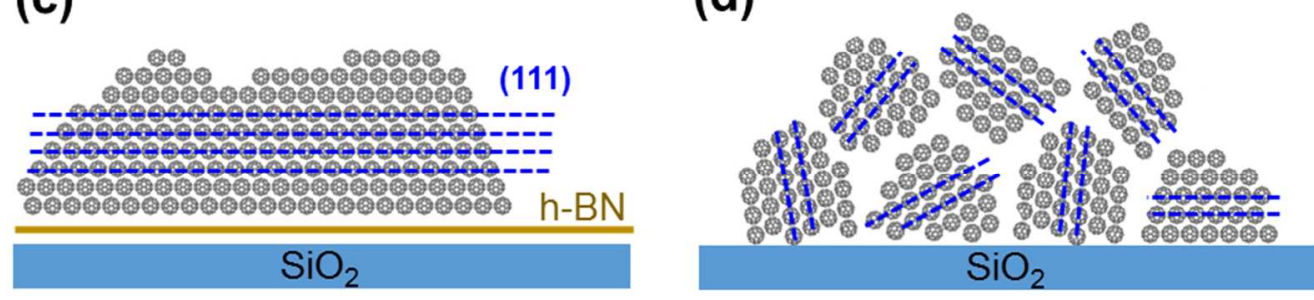

Figure 3. Well-ordered $\mathrm{C}_{60}$ grains on $\mathrm{CVD}$ h-BN compared to $\mathbf{C}_{60}$ on $\mathrm{SiO}_{2}$. (a) GIXD measurement data for $\mathrm{C}_{60}$ on CVD h-BN. The diffraction peaks on the meridian at $\mathrm{q}_{\mathrm{z}}=0.76 \AA^{-1}$ show that (111) planes of the $\mathrm{C}_{60}$ face-centered-cubic crystals are well-ordered and parallel to the h-BN surface. (b) GIXD measurement for $\mathrm{C}_{60}$ on $\mathrm{SiO}_{2}$. The ring shaped diffraction patterns indicates that $\mathrm{C}_{60}$ grains on $\mathrm{SiO}_{2}$ are randomly oriented in all directions. (c) $\mathrm{C}_{60}$ packing structure on h-BN. (d) $\mathrm{C}_{60}$ packing structure on $\mathrm{SiO}_{2}$. 

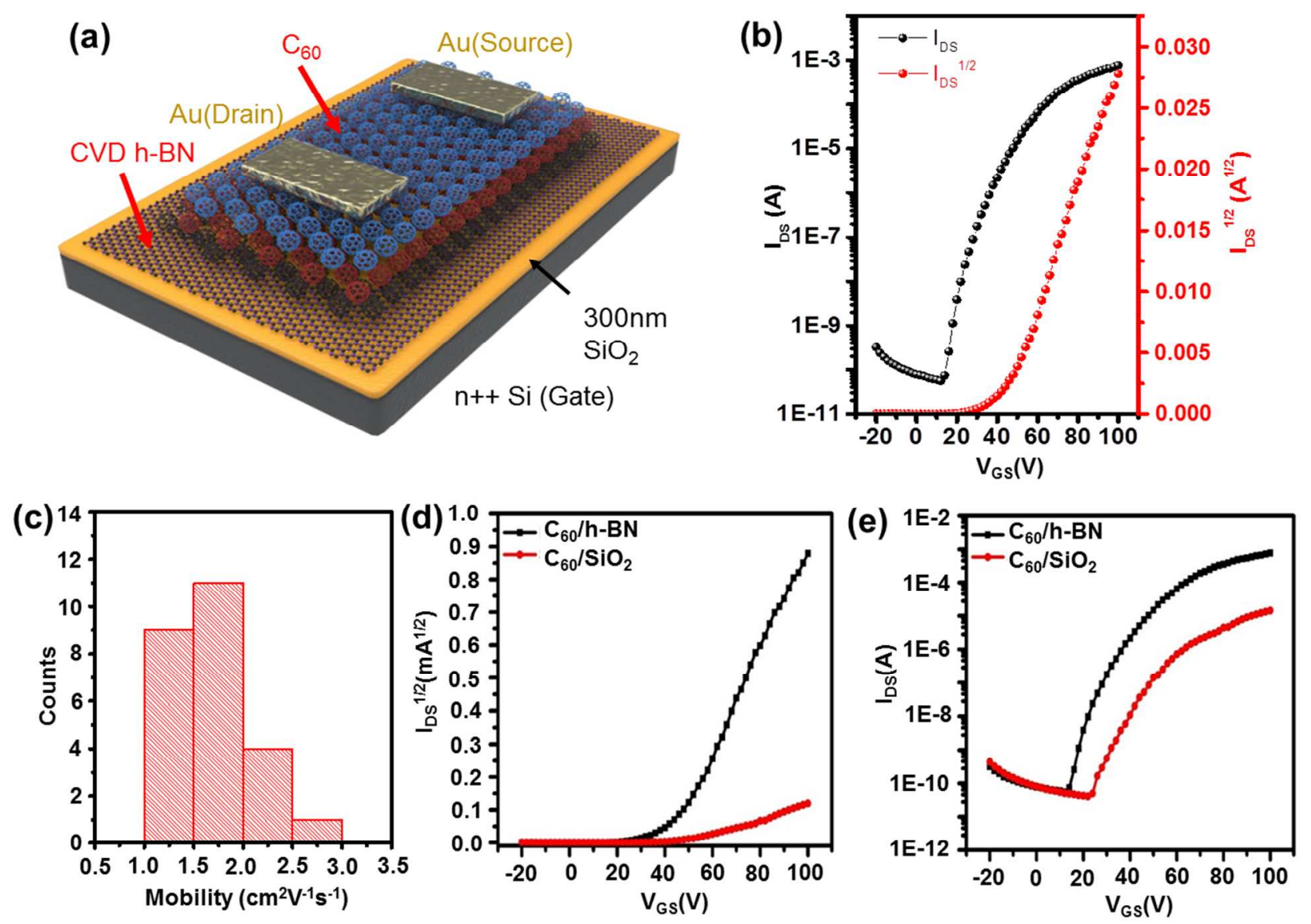

Figure 4. Electrical characteristics of $\mathbf{C}_{60}$ FETs on CVD h-BN. (a) Schematics of $\mathrm{C}_{60}$ FET on CVD h-BN. (b) Transfer curve of $\mathrm{C}_{60}$ FETs on h-BN with the maximum mobility of $2.9 \mathrm{~cm}^{2} \mathrm{~V}^{-1} \mathrm{~s}^{-}$ 1. (c) Histogram of electron mobility measured in $\mathrm{C}_{60}$ FETs on h-BN. The average mobility is around $1.7 \mathrm{~cm}^{2} \mathrm{~V}^{-1} \mathrm{~s}^{-1}$. (d,e) Comparison of transfer curves from $\mathrm{C}_{60}$ FETs on h-BN vs. on $\mathrm{SiO}_{2}$. Panel d shows the $\mathrm{I}_{\mathrm{DS}}{ }^{1 / 2}$ data on a linear scale. Panel e shows the $\mathrm{I}_{\mathrm{DS}}$ data on a semi-log scale. 


\section{ASSOCIATED CONTENT}

Supporting Information. Additional adsorption energy calculations of $\mathrm{C}_{60}$ on h-BN with detailed calculation methods, additional AFM and TEM images of samples, TEM data on $\mathrm{C}_{60}$ epitaxy on mechanically exfoliated h-BN flakes, and output curves of $\mathrm{C}_{60} / \mathrm{h}-\mathrm{BN}$ devices. This material is available free of charge via the Internet at http://pubs.acs.org.

\section{AUTHOR INFORMATION}

$\uparrow$ These authors contributed equally to this work.

\section{Corresponding Author}

*Address correspondence to Y.N. (nishiy@stanford.edu) or Z.B. (bao@stanford.edu)

\section{ACKNOWLEDGMENT}

T.H.L. is supported by a Research Grant from Kwangwoon University in 2016 and by Stanford CIS-FMA program and ILJU Foundation. K.K. acknowledges the support by the FutureInnovative Research Fund (1.170005.01) of UNIST (Ulsan National Institute of Science \& Technology) and Nano·Material Technology Development Program through the National Research Foundation of Korea(NRF) funded by the Ministry of Science, ICT and Future Planning.(2009-0082580) H.J.P. and Z.L. acknowledge the support by the National Research Foundation of Korea (NRF) grant funded by the Korea government (MSIP) (No. 2015R1A2A2A01006992). D.S. thanks the PhD studentship from the EPSRC-DTP award. L.S. gratefully acknowledges support from the Kodak Graduate Fellowship. E.J.G.S. acknowledges the use of computational resources from the UK national high performance computing service, ARCHER, for which access was obtained via the UKCP consortium and funded by EPSRC grant 
ref EP/K013564/1; and the Extreme Science and Engineering Discovery Environment (XSEDE), supported by NSF grants number TG-DMR120049 and TG-DMR150017. The Queen's Fellow Award through the startup grant number M8407MPH as well as the Energy PRP funded by Queen's University Belfast are also acknowledged. X.G. acknowledges support through the Bridging Research Interactions through collaborative the Development Grants in Energy (BRIDGE) program under the SunShot initiative of the Department of Energy program under contract DE-FOA-0000654-1588. ZB acknowledges support from the National Science Foundation (grant no. DMR-1303178). Portions of this research were carried out at the Stanford Synchrotron Radiation Lightsource, a national user facility operated by Stanford University on behalf of the US DOE, Office of Basic Energy Sciences under Contract No. DE-AC02$76 \mathrm{SF} 00515$.

\section{REFERENCES}

1. Arias, A. C.; Mackenzie, J. D.; Mcculloch, I.; Rivnay, J.; Salleo, A. Materials and Applications for Large Area Electronics: Solution-Based Approaches. Chem. Rev. 2010, 110, 324.

2. Klauk, H. Organic Thin-Film Transistors. Chem. Soc. Rev. 2010, 39, 2643-2666.

3. Di Carlo, A.; Piacenza, F.; Bolognesi, A.; Stadlober, B.; Maresch, H. Influence of Grain Sizes on the Mobility of Organic Thin-Film Transistors. Appl. Phys. Lett. 2005, 86, 263501.

4. Di, C.-a.; Liu, Y.; Yu, G.; Zhu, D. Interface Engineering: An Effective Approach toward High-Performance Organic Field-Effect Transistors. Acc. Chem. Res. 2009, 42, 1573-1583.

5. Jurchescu, O. D.; Popinciuc, M.; Van Wees, B. J.; Palstra, T. T. M. Interface-Controlled, High-Mobility Organic Transistors. Adv. Mater. 2007, 19, 688-692. 
6. Singh, T. B.; Sariciftci, N. S.; Yang, H.; Yang, L.; Plochberger, B.; Sitter, H. Correlation of Crystalline and Structural Properties of Thin Films Grown at Various Temperature with Charge Carrier Mobility. Appl. Phys. Lett. 2007, 90, 213512.

7. Diao, Y.; Tee, B. C. K.; Giri, G.; Xu, J.; Kim, D. H.; Becerril, H. A.; Stoltenberg, R. M.; Lee, T. H.; Xue, G.; Mannsfeld, S. C. B.; Bao, Z. Solution Coating of Large-Area Organic Semiconductor Thin Films with Aligned Single-Crystalline Domains. Nat. Mater. 2013, 12, 665671.

8. Horowitz, G.; Hajlaoui, M. E. Grain Size Dependent Mobility in Polycrystalline Organic Field-Effect Transistors. Synth. Met. 2001, 122, 185-189.

9. Lee, C.-H.; Schiros, T.; Santos, E. J. G.; Kim, B.; Yager, K. G.; Kang, S. J.; Lee, S.; Yu, J.; Watanabe, K.; Taniguchi, T.; Hone, J.; Kaxiras, E.; Nuckolls, C.; Kim, P. Epitaxial Growth of Molecular Crystals on Van Der Waals Substrates for High-Performance Organic Electronics. Adv. Mater. 2014, 26, 2812-2817.

10. Kang, S. J.; Lee, G.-H.; Yu, Y.-J.; Zhao, Y.; Kim, B.; Watanabe, K.; Taniguchi, T.; Hone, J.; Kim, P.; Nuckolls, C. Organic Field Effect Transistors Based on Graphene and Hexagonal Boron Nitride Heterostructures. Adv. Funct. Mater. 2014, 24, 5157-5163.

11. He, D.; Zhang, Y.; Wu, Q.; Xu, R.; Nan, H.; Liu, J.; Yao, J.; Wang, Z.; Yuan, S.; Li, Y.; Shi, Y.; Wang, J.; Ni, Z.; He, L.; Miao, F.; Song, F.; Xu, H.; Watanabe, K.; Taniguchi, T.; Xu, J.-B.; Wang, X. Two-Dimensional Quasi-Freestanding Molecular Crystals for High-Performance Organic Field-Effect Transistors. Nat. Commun. 2014, 5, 5162.

12. Kim, K.; Santos, E. J. G.; Lee, T. H.; Nishi, Y.; Bao, Z. Epitaxially Grown Strained Pentacene Thin Film on Graphene. Small 2015, 11, 2037-2043. 
13. Kim, K.; Lee, T. H.; Santos, E. J. G.; Jo, P. S.; Salleo, A.; Nishi, Y.; Bao, Z. Structural and Electrical Investigation of $\mathrm{C}_{60}$-Graphene Vertical Heterostructures. ACS Nano 2015, 9, 59225928.

14. Lee, W. H.; Park, J.; Sim, S. H.; Lim, S.; Kim, K. S.; Hong, B. H.; Cho, K. SurfaceDirected Molecular Assembly of Pentacene on Monolayer Graphene for High-Performance Organic Transistors. J. Am. Chem. Soc. 2011, 133, 4447-4454.

15. Hlaing, H.; Kim, C.-H.; Carta, F.; Nam, C.-Y.; Barton, R.; Petrone, N.; Hone, J.; Kymissis, I. Low-Voltage Organic Electronics Based on a Gate-Tunable Injection Barrier in Vertical Graphene-Organic Semiconductor Heterostructures. Nano Lett. 2015, 15, 69-74.

16. Dean, C. R.; Young, A. F.; Meric, I.; Lee, C.; Wang, L.; Sorgenfrei, S.; Watanabe, K.; Taniguchi, T.; Kim, P.; Shepard, K. L.; Hone, J. Boron Nitride Substrates for High-Quality Graphene Electronics. Nat. Nanotechnol. 2010, 5, 722-726.

17. Xue, J.; Sanchez-Yamagishi, J.; Bulmash, D.; Jacquod, P.; Deshpande, A.; Watanabe, K.; Taniguchi, T.; Jarillo-Herrero, P.; Leroy, B. J. Scanning Tunnelling Microscopy and Spectroscopy of Ultra-Flat Graphene on Hexagonal Boron Nitride. Nat. Mater. 2011, 10, 282285.

18. Decker, R.; Wang, Y.; Brar, V. W.; Regan, W.; Tsai, H. Z.; Wu, Q.; Gannett, W.; Zettl, A.; Crommie, M. F. Local Electronic Properties of Graphene on a BN Substrate via Scanning Tunneling Microscopy. Nano Lett. 2011, 11, 2291-2295.

19. Gannett, W.; Regan, W.; Watanabe, K.; Taniguchi, T.; Crommie, M. F.; Zettl, A. Boron Nitride Substrates for High Mobility Chemical Vapor Deposited Graphene. Appl. Phys. Lett. 2011, 98, 242105. 
20. Song, L.; Ci, L.; Lu, H.; Sorokin, P. B.; Jin, C.; Ni, J.; Kvashnin, A. G.; Kvashnin, D. G.; Lou, J.; Yakobson, B. I.; Ajayan, P. M. Large Scale Growth and Characterization of Atomic Hexagonal Boron Nitride Layers. Nano Lett. 2010, 10, 3209-3215.

21. Kim, K. K.; Hsu, A.; Jia, X.; Kim, S. M.; Shi, Y.; Hofmann, M.; Nezich, D.; RodriguezNieva, J. F.; Dresselhaus, M.; Palacios, T.; Kong, J. Synthesis of Monolayer Hexagonal Boron Nitride on Cu Foil Using Chemical Vapor Deposition. Nano Lett. 2012, 12, 161-166.

22. Kim, G.; Jang, A. R.; Jeong, H. Y.; Lee, Z.; Kang, D. J.; Shin, H. S. Growth of HighCrystalline, Single-Layer Hexagonal Boron Nitride on Recyclable Platinum Foil. Nano Lett. 2013, 13, 1834-1839.

23. Schulz, F.; Drost, R.; Hämäläinen, S. K.; Liljeroth, P. Templated Self-Assembly and Local Doping of Molecules on Epitaxial Hexagonal Boron Nitride. ACS Nano 2013, 7, 1112111128.

24. Joshi, S.; Bischoff, F.; Koitz, R.; Ecija, D.; Seufert, K.; Seitsonen, A. P.; Hutter, J.; Diller, K.; Urgel, J. I.; Sachdev, H.; Barth, J. V.; Auwärter, W. Control of Molecular Organization and Energy Level Alignment by an Electronically Nanopatterned Boron Nitride Template. ACS Nano 2014, 8, 430-442.

25. Urgel, J. I.; Schwarz, M.; Garnica, M.; Stassen, D.; Bonifazi, D.; Ecija, D.; Barth, J. V.; Auwärter, W. Controlling Coordination Reactions and Assembly on a $\mathrm{Cu}(111)$ Supported Boron Nitride Monolayer. J. Am. Chem. Soc. 2015, 137, 2420-2423.

26. Mickelson, W.; Aloni, S.; Han, W. Q.; Cumings, J.; Zettl, A. Packing $\mathrm{C}_{60}$ in Boron Nitride Nanotubes. Science 2003, 300, 467-469. 
27. Hinderhofer, A.; Gerlach, A.; Broch, K.; Hosokai, T.; Yonezawa, K.; Kato, K.; Kera, S.; Ueno, N.; Schreiber, F. Geometric and Electronic Structure of Templated $\mathrm{C}_{60}$ on Diindenoperylene Thin Films. J. Phys. Chem. C 2013, 117, 1053-1058.

28. Smilgies, D.-M. Scherrer Grain-Size Analysis Adapted to Grazing-Incidence Scattering with Area Detectors. J. Appl. Cryst. 2009, 42, 1030-1034.

29. Singh, T. B.; Marjanović, N.; Matt, G. J.; Günes, S.; Sariciftci, N. S.; Montaigne Ramil, A.; Andreev, A.; Sitter, H.; Schwödiauer, R.; Bauer, S. High-Mobility N-Channel Organic FieldEffect Transistors Based on Epitaxially Grown $\mathrm{C}_{60}$ Films. Organic Electronics 2005, 6, 105-110.

30. Itaka, K.; Yamashiro, M.; Yamaguchi, J.; Haemori, M.; Yaginuma, S.; Matsumoto, Y.; Kondo, M.; Koinuma, H. High-Mobility C60 Field-Effect Transistors Fabricated on MolecularWetting Controlled Substrates. Adv. Mater. 2006, 18, 1713-1716.

31. Virkar, A.; Mannsfeld, S.; Oh, J. H.; Toney, M. F.; Tan, Y. H.; Liu, G.-y.; Scott, J. C.; Miller, R.; Bao, Z. The Role of Ots Density on Pentacene and $\mathrm{C}_{60}$ Nucleation, Thin Film Growth, and Transistor Performance. Adv. Funct. Mater. 2009, 19, 1962-1970.

32. Li, H.; Tee, B. C. K.; Cha, J. J.; Cui, Y.; Chung, J. W.; Lee, S. Y.; Bao, Z. High-Mobility Field-Effect Transistors from Large-Area Solution-Grown Aligned $\mathrm{C}_{60}$ Single Crystals. J. Am. Chem. Soc. 2012, 134, 2760-2765.

33. Regan, W.; Alem, N.; Aleman, B.; Geng, B. S.; Girit, C.; Maserati, L.; Wang, F.; Crommie, M.; Zettl, A. A Direct Transfer of Layer-Area Graphene. Appl. Phys. Lett. 2010, 96, 113102.

34. José, M. S.; Emilio, A.; Julian, D. G.; Alberto, G.; Javier, J.; Pablo, O.; Daniel, S.-P. The Siesta Method for Ab Initio Order- N Materials Simulation. J. Phys.: Condens. Matter 2002, 14, 2745 . 
35. Kresse, G.; Hafner, J. Ab Initio Molecular Dynamics for Open-Shell Transition Metals. Phys. Rev. B 1993, 48, 13115-13118.

36. Kresse, G.; Furthmüller, J. Efficient Iterative Schemes for Ab Initio Total-Energy Calculations Using a Plane-Wave Basis Set. Phys. Rev. B 1996, 54, 11169-11186.

37. Perdew, J. P.; Burke, K.; Ernzerhof, M. Generalized Gradient Approximation Made Simple. Phys. Rev. Lett. 1996, 77, 3865-3868.

38. Jiří, K.; David, R. B.; Angelos, M. Chemical Accuracy for the Van Der Waals Density Functional. J. Phys.: Condens. Matter 2010, 22, 022201.

39. Thonhauser, T.; Cooper, V. R.; Li, S.; Puzder, A.; Hyldgaard, P.; Langreth, D. C. Van Der Waals Density Functional: Self-Consistent Potential and the Nature of the Van Der Waals Bond. Phys. Rev. B 2007, 76, 125112. 

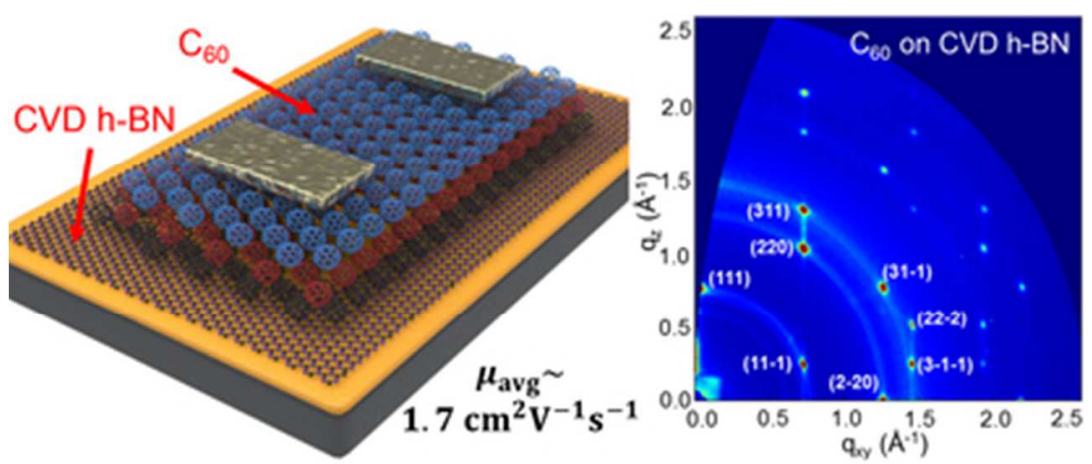

TOC Graphic

$34 \times 14 \mathrm{~mm}(300 \times 300$ DPI $)$ 